Review Article

\title{
Radiation induced caries: An overview
}

\section{Mithra N Hegde ${ }^{1}$, Ananiya John ${ }^{2}$}

${ }^{1}$ Head of the Department, ${ }^{2}$ Post Graduate Student, Department of Conservative Dentistry and Endodontics, A B Shetty Memorial institute of Dental Sciences, Nitte deemed to be university, Mangaluru, Karnataka, India.

Corresponding Author: Mithra N Hegde, Head of the Department, Department of Conservative Dentistry and Endodontics, A B Shetty Memorial institute of Dental Sciences, Nitte deemed to be university, Mangaluru, Karnataka, India.

Mobile :+919845284411 E-mail:drhegdedentist@gmail.com

Received

: 02.11.2018

Review Completed : 12.11.2018

Accepted

: 15.11 .2018

Keywords : Radiotherapy, caries, head and neck cancer, xerostomia, fluoride

\begin{tabular}{|c|}
\hline Access this article online \\
\hline Quick Response Code \\
\hline
\end{tabular}

\begin{abstract}
:
Amongst all the different varieties of cancers diagnosed across the world around $6 \%$ is found to be head and neck cancer and radiotherapy is the choice of treatment. Head and neck radiotherapy cause several changes in dentition, saliva, oral microflora and food habits of the patient thus increasing the risk of radiation-induced dental caries a common, yet serious complication. This paper analyses the literature pertaining to radiation-induced caries and discusses the predisposing factors, etiology, structural changes in the tooth, clinical features of radiation caries and management. To review this, the Pubmed and Scopus database was searched using the keywords "radiation-induced caries", "xerostomia", "effects of radiology" and "head and neck tumors", "osteoradionecrosis", "management of radiation caries", "structural changes in teeth after radiotherapy", "prevention of radiation caries". Radiation caries is a rampant form of caries commonly seen within the first 3 months after the radiotherapy. Thus, early dynamic involvement of the dentist in the preventive and therapeutic strategies are important. Furthermore, educating and motivating patients about the risk factors, maintenance of oral hygiene, stimulation of salivary flow, fluoride use, and nutritiona orientation are essential to decrease the incidence of radiation caries and eventually enhances the quality of life of patients.
\end{abstract}

\section{Introduction}

Head and neck radiotherapy is very common treatment modality in today's scenario due to the increased prevalence of cancer and ionizing radiation is the therapeutic agent used.[1]This technique semi selectively damages the genetic material of vulnerable malignant cells, straight or through the production of free radicals, which leads to cell death. However, besides the therapeutic anti-cancer effect, the ionizing irradiation can also harm healthy and normal tissues, especially cells involved in the rapid division[2] which are located near to radiation field, ultimately ends in both acute and chronic side effects.[3]

This results in various complications in the oral cavity which affects salivary glands, oral mucous membrane, bone, masticatory musculature, and dentition.[4] The main post radiation diseases are mucositis, xerostomia, dental caries, dysaesthesia, bacterial and fungal infection, trismus, and osteoradionecrosis.[5] One of the initial difficulties after radiotherapy is the initiation of abnormal caries known as radiation caries.[6] Radiation caries is a highly destructive form of dental caries.[7,8] They are commonly seen after the first 3 months of radiotherapy.[9] They are rapid in onset with quick progress, most dangerous cases may lead to complete destruction of normal healthy dentition within a year, disturbing the patient's quality of life.[10]Even in the advanced cases pain as such will be absent.[8]

\section{Material and methods}

The present study was done to find the updated information on radiation caries. A search was made using the keywords "radiation-induced caries", "xerostomia", "effects of radiology" and "head and neck tumors", "osteoradionecrosis", "management of radiation caries", "structural changes in teeth after radiotherapy", "prevention of radiation caries", etc in Pubmed and Scopus. Later the results were refined by adding filters based on time duration (1991-2018) Around 40 relevant articles 
were selected from the searched data. And Crossreferencing identified additionally relevant studies.

\section{Predisposing factors}

The clinical type and severity depends on the following factors

1. Original vulnerability to caries: Greater pre-irradiation caries, more the susceptibility to post radiation caries.

2. Exposure of cementum with the gingival recession: If the cementum is exposed, the possibility of development of cervical caries will be more in irradiated patients.

3. Oral hygiene status: Xerostomia results in plaque formation due to the reduced presence and anticariogenic efficacy of saliva and thus initiate caries of all surfaces. If the oral hygiene status is poor, it increases the pace and extent of caries.

4. Changes in the diet: Because of uneasiness caused by mucositis, patients shift from a coarse detergent diet to a soft adhesive one. The high content of carbohydrate in soft diets produces heavy plaque. Plaque rich in carbohydrate, along with xerostomia correlate with a high cariogenic potential.[11]

\section{Etiology}

Age of the patient, original radiation dose, extent of radiation exposure field which is assessed by the location and actual size of a tumor, patient's general health condition and supplementary risk factors like alcoholism, smoking, and malnourishment contributes to the severity and occurrence of radiation-induced changes in the oral cavity.[12]

1. Change in the quantity, quality, and composition of saliva

Radiotherapy alters the quantity, quality, and composition of saliva, leading to the reduced flow of saliva, buffering capacity, levels of electrolyte and immunoprotein, oral clearance and an increase in acidity and levels of cariogenic bacteria.[13] Even a low dose of 20 Gy can result in changes in the amount of saliva and its consistency. Saliva changes to sparse, thick and ropy after 4-5 fractions[14] subsequently weakens the self-cleaning of the teeth. All of these together contribute to hyposalivation in major and minor salivary glands and leads to related changes in microbial, chemical, immunologic, and dietary parameters of cariogenicity contributing to a huge rise in caries in patients undergoing radiotherapy.[15]

\section{Changes in taste perception increasing the need for} highly flavored foods

As an outcome of radiation on the taste corpuscles, a change of taste is seen after the radiation with a reduction of $50 \%$ in the perception of bitter and acid tastes. Due to the loss of pleasure in eating patients tends to eat highly flavored food. Taste buds of fungiform and circumvallate papillae are very sensitive to radiation resulting in partial or total loss of taste.[16]

\section{Fluctuations in nutritional status demanding consumption of highly calorific foods}

\section{Ecological changes to oral micro biota}

The amount of cariogenic bacteria, i.e. Lactobacillus and Streptococcus mutans, rises in the saliva, and so does the level of immunoglobulin A (IgAs). These changes are seen from the beginning of radiotherapy up to three months after its completion and seen to noticeably increase the risk of radiation caries.[17] Furthermore, the population of Actinomyces naeslundi, seen in periodontal disease and root caries, also increases after radiation.[18]

\section{Radiation mucositis as a probable reason}

Radiation mucositis is the reactive inflammation of the oral and oropharyngeal mucous membranes as the first consequence of radiation therapy. Activities like drinking, eating, swallowing and speech will be discomfort able and painful.[8] Oral hygiene habits of such patients will be compromised due to the additional pain associated with tooth brushing ultimately increasing the risk of radiation caries.

\section{Changes in the dental hard tissues structure}

Microscopic structural changes will occur in enamel, dentine, cementum, and DEJ which makes the tooth more prone to caries by increasing their susceptibility to 
demineralization.[19] As a result of changes in $\mathrm{pH}$ and buffering capacity of saliva, minerals present in enamel and dentin will dissolve easily. Hence, process of remineralization of the dental hard tissue is hampered. [17] These two mechanisms increase the risk of caries.

\section{Truisms and general malaise}

Due to trismus and malaise patients find it difficult to maintain oral hygiene practices leading to radiation caries.[20] Trismus is due to hypovascularization and fibrosis of the masticatory or temporomandibular muscles tissues after the radiotherapy.[21]

\section{Microscopic changes}

Even a low dose of $0.50 \mathrm{~Gy}$ of high energy x-ray radiation is enough for decreasing the mechanical properties in enamel and dentin.[22]

\section{Enamel}

The initial damage from irradiation occurs in organic portion of the enamel, that is, in the interprismatic space. Water content will be reduced which causes alternations in the mechanical properties of enamel. These water molecules will get oxidized to hydrogen peroxide and hydrogen free radicals which subsequently denature the organic components leading to impaired mechanical properties and integrity of the enamel. Changes in the inorganic structures of enamel are also reported.[23]

\section{Dentin}

Microhardness and stability of dentin are reduced due to irradiation which results in changes in organic components within the dentinal tubules. Due to the presence of water free radicals, hydrogen peroxide will be formed as a function of radiation, hence this will denature the organic content of dentin, thus lessening the internal stability of the tissue.[22]

\section{DEJ}

Odontoblast process extends well into the enamel in the normal tooth but in an irradiated tooth it stops in front of DEJ. Similarly, the anchoring between dentin and enamel are also affected by creating gaps between them. Even the contours of the DEJ changes from sharp to diffuse and damaged type as a result of radiation.[22]

\section{Clinical features}

Radiation caries are a highly destructive form of dental caries.[7,8] They are commonly seen after the first 3 months of radiotherapy.[9] They are rapid in onset with quick progress, most dangerous cases may lead to complete destruction of normal healthy dentition within a year. These lesions are commonly seen on the smooth surfaces of the incisors, premolars, and molars which are most resistant to caries among normal individuals due to the reduction in the continues supply of saliva from the salivary glands.[8]

Clinically 3 patters of radiation caries are commonly seen.

\section{Type 1}

Radiation Caries is set around the necks of the teeth, which usually encircles the cervical area completely hence named as caries circularis. [8] It initiates on the labial surface of the incisors and canines superficially and later extended to complete cervical area of the crown and then progresses to the inner surface, resulting in the total amputation of the crown.This type is less frequently in molars.[24]

\section{Type 2}

Lesion initiates as a spot depression spreading from the incisal or occlusal edges on the labial or buccal and lingual surfaces. During the progression, enamel shell becomes destroyed and coronal dentin becomes partially disintegrated.[24]

\section{Type 3}

Type 3 is seen as brown to black discoloration of the crown. These are commonly superficial defects affecting the buccal and later the lingual or palatal surfaces of the tooth crown. The proximal surfaces are affected less. This type of caries starts as a diffuse, punctate defect and progresses to generalized, irregular erosion of the tooth surfaces. The decay is superficial and restricted to incisal or occlusal edges causing the damage of the coronal enamel and dentin.[24] 


\section{Management}

\section{Pre-radiation}

\section{Dental awareness and assessment}

One of the most important responsibility of a dentist is to make the patient aware of the adverse effects in the oral cavity after a head and neck radiotherapy. They should be conscious as well as self motivated to minimize potentially disturbing and endangering dental complications.[25] A complete dental examination including clinical examination of soft tissue and hard tissue, the status of oral hygiene should be done. Full mouth radiographs must be taken and evaluation of teeth including vitality, recontouring of restorations, the presence of carious lesions, need of endodontic therapy should be done.[26]

\section{Prevention of xerostomia}

Xerostomia is the foremost reason for the development of radiation caries due to change in the volume, consistency, and $\mathrm{pH}$ of secreted saliva. The severity of xerostomia depends on total radiation dose and on the volume of irradiated tissue.[27] As per American Cancer Society Head and Neck, Cancer Survivorship Care Guidelines to prevent xerostomia, alcohol-free rinses should be encouraged in head and neck cancer survivor as it rehydrates the mucosa, neutralize $\mathrm{pH}$, break-up mucus, and prevent crusting of the oral mucosa. It is also recommended for head and neck cancer survivors to have a low-sugar diet and to refrain from caffeine, spicy and highly acidic foods, and tobacco and drinking of fluoridated tap water to prevent dehydration even though xerostomia will not be completely eliminated.[28]Brennan et al (the American Society of Clinical Oncology) suggested the use of salivary gland sparing RT, cytoprotective agents, preservation by stimulation with cholinergic muscarinic agonists (pilocarpine, cevimeline), and the surgical transfer of submandibular glands to prevent xerostomia.[29]

Stem cell replacement therapy are good choice for radiotherapy induced hyposalivation, but an advanced research is still needed.[30]

\section{Restorative management}

Before the beginning of radiotherapy carious teeth and fractured teeth should be restored preferably using GIC due to the fluoride-releasing property of the material, hence reducing recurrent caries.[31] Smoothing or repair of sharp cusps or restoration is mandatory as they may cause trauma to the vulnerable irradiated soft tissues.[32]

\section{Extractions}

Dental extractions after radiotherapy should be avoided as it increases the risk of osteoradionecrosis.[26] According to the Ben-David et al. criteria for extractions includes nonrestorable caries, or caries covering the gum line, teeth with large, compromised restorations with significant loss of attachment (pocketing $>5 \mathrm{~mm}$ ), and teeth with severe erosion or abrasion are extracted if they are in target region.[33]

\section{During radiation}

\section{Oral hygiene}

Daily brushing using soft toothbrush which reaching to all the contours and gingival crevices of the teeth is important to maintain oral hygiene.[34] Fluoridated toothpaste which prevents caries and toothpaste with natural enzymes which reduce the bacterial load on dentition can be used.[34,35] Flossing should be done with unwaxed floss.[36] Sodium bicarbonate rinses are used broadly to raise salivary $\mathrm{pH}$ and buffering capacity can be used.[34]

Topical fluoride applications are important in the prevention of radiation-induced caries. Recommended fluoride preparations are 0.4 percent stannous fluoride, 1.23 percent sodium fluoride or 1.23 percent acidulated phosphate fluoride. Brush on technique or customized tray can be used [34] for a minimum of 5-10 minutes once daily. The patient should not rinse, drink, eat or brush for the following 30 minutes after fluoride application. [35] Studies have shown that combinations of chlorhexidine and fluoride produced good results in controlling radiationinduced caries.[34] Studies have reported that using a regimen of four topical applications of 1.0 sodium fluoride$1 \%$ chlorhexidine solution, and daily rinses with a $0.05 \%$ sodium fluoride- $0.2 \%$ chlorhexidine solution, could achieve complete prevention of radiation caries and remineralization of incipient caries.[35] 


\section{Diet modification}

In order to prevent radiation caries refined carbohydrates must be substituted with constituents such as sorbitol, xylitol, aspartame and saccharine which are not degraded into organic acids by oral bacteria. Dieticians prefer Frequent small meals with high calories foods. Oral cavity should be properly rinsed and brushed after every meal.[37]

\section{Post-radiation}

Frequent follow up appointments should be scheduled and the oral cavity should be properly assessed even after the radiotherapy in all appointments to find any complications or presence of radiation-induced caries. Oral prophylaxis is unavoidable with regular scaling and polishing under antibiotic coverage if oral hygiene is not proper. Extractions are not the treatment of choice after irradiation and must be avoided preferably and endodontic therapy is the better choice. Carious lesions should be restored using GIC without delay.[38] To prevent recurrent caries in irradiated patients oral hygiene and diet modifications should be properly continued as before throughout life. McComb et al. [39] confirmed the efficiency of fluoride releasing materials in the control of recurrent caries in irradiated

\section{References}

1. VissinkA, Jansma J, Spijkervet FK, Burlage FR, Coppes RP. Oral sequelae of head and neck radiotherapy. Crit Rev Oral Biol Med.2003; 14(3):199-212.

2. Beech N, Robinson S, Porceddu S, Batstone M. Dental management of patients irradiated for head and neck cancer. Aust Dent J 2014;59:208.

3. Nutting CM, Morden JP, Harrington KJ, et al. Parotid-sparing intensity modulated versus conventional radiotherapy in head and neck cancer (PARSPORT): a phase 3 multicentre randomised controlled trial. Lancet Oncol 2011;12:127-136.

4. Kielbassa AM, Hinkelbein W, Hellwig E, Meyer-Lückel H. Radiationrelated damage to dentition. The lancet oncology. $2006 \mathrm{Apr}$ 1;7(4):326-35.

5. Naidu MU, Ramana GV, Rani PU, Mohan IK, Suman A, Roy P. Chemotherapy-induced and/or radiation therapy-induced oral mucositis-complicating the treatment of cancer. Neoplasia 2004;6:423-31.

6. Fattore L, Rosenstein HE, Fine L. Dental rehabilitation of the patient with severe caries after radiation therapy. Spec Care Dentist 1986;6:258-61.

7. Dreizen S, Brown LR, Daly TE, Drane JB. Prevention of xerostomiarelated dental caries in irradiated cancer patients. J Dent Res 1977;56:99-104.

8. Kielbassa AM, Hinkelbein W, Hellwig E, Meyer-Lückel H. Radiationrelated damage to dentition. Lancet Oncol 2006; 7:326-335.

9. Lopes MA, Coletta RD, Alves FA, Abbade N, Rossi Junior A Reconhecendo e controlandoosefeitoscolaterais da radioterapia. Rev patients. Hu et al.[40] showed glass ionomers can prevent the formation of secondary caries, even after the loss of restoration.

\section{Conclusion}

Radiation therapy is a widely used resource to treat cancer. Because it is an unspecific therapy, however, nonneoplastic cells are also affected by the treatment. A careful, constant and thorough understanding of the complications of radiotherapy is necessary in order to undertake measures in every phase of treatment. Radiation caries has multifactorial etiology, but hyposalivation remains the primary cause. For an effective prevention and treatment of radiation caries, dental professionals along with oncologists should perform a teamwork right from the treatment planning till the followups. Moreover, motivating the patients to follow proper oral hygiene, stimulation of salivary flow using different aids available, the importance of fluoride use, and nutritional orientation is vital to cut the incidence as well as post-radiation complications of radiation caries and eventually improve the well being of head and neck cancer patients.

Assoc Paul Cir Dent. 1998;52(3):241-4.Basu T, Laskar SG, Gupta T, Budrukkar A, Murthy V, Agarwal JP. Toxicity with radiotherapy for oral cancers and its management: a practical approach. J Cancer Res Ther.2012; 8(2): S72-84.

10. DobrosK, Hajto-Bryk J, Wroblewska M, Zarzecka J. Radiation-induced caries as the late effect of radiation therapy in the head and neck region. Contemporary Oncology. 2016;20(4):287.

11. Hayward JR, Kerr DA, Jesse RH, Castigliano SG, Lampe I, Ingle JI. The management of teeth related to the treatment of oral cancer. CA Cancer J Clin. 1969; 19(2):98-106.

12. Dobros K, Hajto-Bryk J, Wroblewska M, Zarzecka J. Radiation-induced caries as the late effect of radiation therapy in the head and neck region. Contemp Oncol (Pozn) 2015; 19 (5): 1-4.

13. Brown LR, Dreizen S, Daly TE, et al. Interrelations of oral microorganisms, immunoglobulins, and dental caries following radiotherapy. J Dent Res 1978;57:882-893.

14. Naidu MU, Ramana GV, Rani PU, Mohan IK, Suman A, Roy P. Chemotherapy-induced and/or radiation therapy-induced oral mucositis-complicating the treatment of cancer. Neoplasia 2004;6:423-31.

15. Vissink A, Jansma J, Spijkervet FK, Burlage FR, Coppes RP. Oral sequelae of head and neck radiotherapy. Crit Rev Oral Biol Med.2003; 14(3):199-212.

16. Goursand D, Borges CM, Alves KM, Birth AM, Winter RR, Martins LHPM, et al. Oral sequelae in children undergoing antineoplastic therapy: causes and definition of the role of dental surgeon. ArqOdontol. 2006; 42 (3): 161-256. 
17. Kielbassa AM, Hinkelbein W, Hellwig E, Meyer-Luckel H. Radiationrelated damage to dentition. Lancet Oncol 2006; 7:326-35.

18. SARI J, Nasiloski KS, GOMES AP. Oral complications in patients receiving head and neck radiation therapy: a literature review. RGORevistaGaúcha de Odontologia. 2014 Dec;62(4):395-400.

19. Pfister DG, Spencer S, Brizel DM, Burtness B, Busse PM, Caudell JJ, et al. Head and neck cancers, Version 2.2014. Clinical practice guidelines in oncology. J Natl ComprCancNetw2014;12:1454-87.

20. Stryjski A, Borysewicz-Lewicka M. Patients oral health status after head and neck neoplasm radiotherapy. Dent Med Probl 2007; 44: 177 83.

21. Wang CJ, Huang EY, Hsu HC, Chen HC, Fang FM, Hsiung CY. The degree and time-course assessment of radiation-induced trismus occurring after radiation therapy for nasopharyngeal cancer. Laryngoscope. 2005;115(8):1458-60

22. Lieshout HF, Bots CP. The effect of radiotherapy on dental hard tissue-a systematic review. Clin Oral Investig. 2014;18(1):17-24.

23. de Siqueira Mellara T, Palma-Dibb RG, de Oliveira HF, Garcia PaulaSilva FW, Nelson-Filho P, da Silva RA et al. The effect of radiation therapy on the mechanical and morphological properties of the enamel and dentin of deciduous teethan in vitro study. Radiat Oncol. 2014; 22; 9:30

24. Rajendran R, Sivapathasundharam B. Shafer's Textbook of Oral Pathology. 7th ed. India: Elsevier Health Sciences; 2012.

25. Andrews N, Griffiths C. Dental complications of head and neck radiotherapy: Part 2. Aust Dent J. 2001;46(3):174-82.

26. Anil S, Philip T, Madhu K, Beena VT, Vijayakumar T. Radiation carries-A rationale approach towards its preventional and management. J Indian Dent Assoc. 1993;64:9-12.

27. Dirix P, Nuyts S, Van den Bogaert W. Radiation-induced xerostomia in patients with head and neck cancer: a literature review. Cancer 2006;107:2525-34.

28. Cohen EE, LaMonte SJ, Erb NL, Beckman KL, Sadeghi N, Hutcheson KA, Stubblefield MD, Abbott DM, Fisher PS, Stein KD, Lyman GH. American Cancer Society head and neck cancer survivorship care guideline. CA: a cancer journal for clinicians. 2016 May;66(3):203-39.

29. Brennan MT, Shariff G, Lockhart PB, Fox PC. Treatment of xerostomia:
A systematic review of therapeutic trials. Dent Clin North Am 2002;46:847-56.

30. Pringle S, Van Os R, Coppes RP. Concise review: Adult salivary gland stem cells and a potential therapy for xerostomia. Stem Cells 2013;31:613-9.

31. Shenoy VK, Shenoy KK, Rodrigues S, Shetty P. Management of oral health in patients irradiated for head and neck cancer: a review. Kathmandu Univ Med J. 2007; 5(1):117-20A

32. Chin DW, Treister N, Friedland B, et al. Effect of dental restorations and prostheses on radiotherapy dose distribution: a Monte Carlo study. J Appl Clin Med Phys 2009;10:2853.

33. Ben-David MA, Diamante M, Radawski JD, et al. Lack of osteoradionecrosis of the mandible after intensity-modulated radiotherapy for head and neck cancer: likely contributions of both dental care and improved dose distributions. Int J Radiat Oncol Bio Phys 2007;68:396-402.

34. Andrews N, Griffiths C. Dental complications of head and neck radiotherapy: Part 2. Aust Dent J. 2001; 46(3):174-82.

35. Chai WL, Ngeow WC, Ramli R, Rahman RA. Managing complications of radiation therapy in head and neck cancer patients: Part II. management of radiation-induced caries. Singapore Dent J.2006; 28(1):4-6.

36. Shenoy VK, Shenoy KK, Rodrigues S, Shetty P. Management of oral health in patients irradiated for head and neck cancer: a review. Kathmandu Univ Med J. 2007; 5(1):117-20

37. Andrews N, Griffiths C. Dental complications of head and neck radiotherapy: Part 2. Aust Dent J. 2001; 46(3):174-82.

38. Kielbassa AM, Schilli K. Betreuung des tumortherapeutischbestrahlten PatientenausSicht der Zahnerhaltung. Zahnärztl Mitt 1997;87:263647.

39. McComb D, Erickson RL, Maxymiw WG, Wood RE. A clinical comparison of glass ionomer, resin-modified glass ionomer and resin composite restorations in the treatment of cervical caries in xerostomic head and neck radiation patients. Oper Dent 2002;27:430-7.

40. Hu JY, Li YQ, Smales RJ, Yip KH. Restoration of teeth with more-viscous glass ionomer cements following radiation-induced caries. Int Dent $J$ 2002;52:445-8. 